\title{
Prevalence and characteristics of shunt malfunction without ventricular size change at King Abdulaziz Medical City in Riyadh
}

Sultan M. Albugami, MBBS, Khalid W. Alwadi, MBBS, Abdulmohhsen K. Alrugaib, MBBS, Abdulrahman M. Alsuwailim, MBBS, Tariq Aljared, MBBS, FRCSC.

\section{ABSTRACT}

فالأهداف : تحديد انتشار العطل في وظيفة التحويلة البطينية الصفاقية فئية

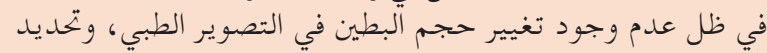

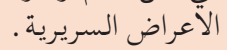

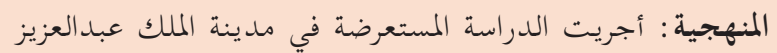

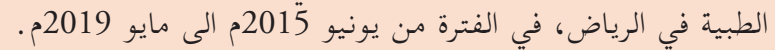

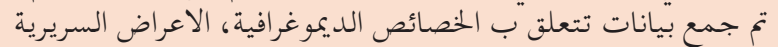

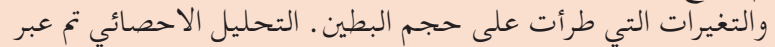
برنامج SPS النسخة الثالثة والعشرون البطن التئ

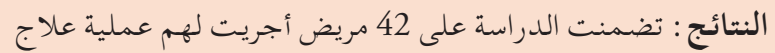

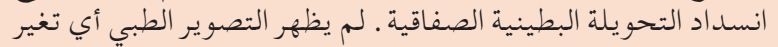

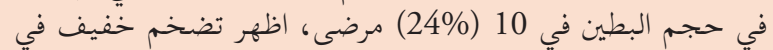

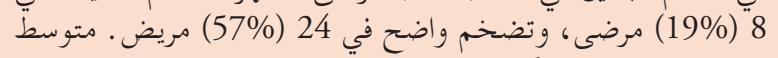

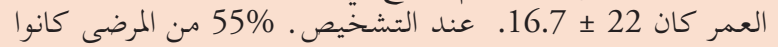

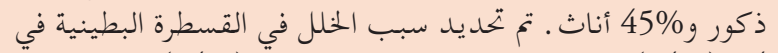

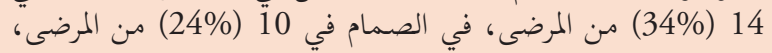

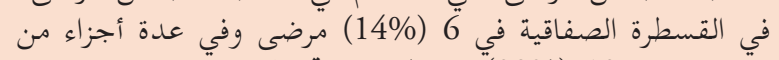

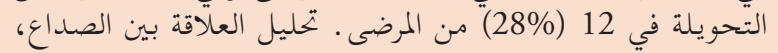

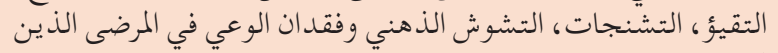

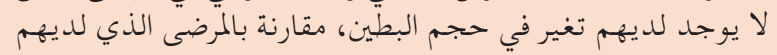

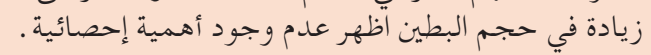

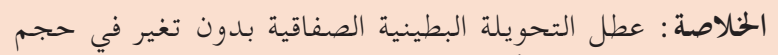

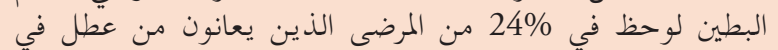

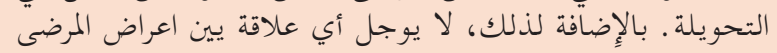

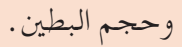

Objectives: To determine the prevalence of shunt malfunction without change in ventricle size in imaging modalities, and its clinical presentation.

Methods: A cross-sectional study conducted at King Abdulaziz Medical City, Riyadh, from June 2015 to May 2019. Patient's demographics, clinical
\end{abstract}

presentation and changes in ventricle size were collected. Statistical analysis was done using SPSS version 23 .

Results: The study included 42 patients who underwent shunt revision. Imaging showed no change in size in $10(24 \%)$ patients, mild enlargement in $8(19 \%)$, and obvious enlargement in $24(57 \%)$. The mean age of diagnosis was $22 \pm 16.7 .55 \%$ of the patients were males, $45 \%$ females. The cause of the malfunction was ventricular catheter occlusion in 14 $(34 \%)$ patients, $10(24 \%)$ patients had valve-related malfunction, and peritoneal catheter occlusion was present in $6(14 \%)$ patients, and $12(28 \%)$ patients had a combination of the previously mentioned causes. Analysis of the association between ventricular size and headache, vomiting, seizure, confusion, and loss of consciousness in patients with unchanged ventricular size and those with increased ventricular size has shown no statistical significance.

Conclusion: Shunt malfunction without ventricular size change was observed in $24 \%$ of all shunt malfunction patients. In addition, there was no relationship between ventricular size and patient symptoms.

Neurosciences 2021; Vol. 26 (1):31-35 doi: 10.17712/nsj.2021.1.20200099

From the Department of pediatric neurosurgery (Aljared), King Abdullah Specialist Children Hospital, from the College of Medicine (Albugami, Alwadi, Alrugaib), King Saud bin Abdulaziz University for Health Sciences, Riyadh, and from the College of Medicine (Alsuwailim), King Faisal University, Al-Ahsa, Kingdom of Saudi Arabia.

Received 12th June 2020. Accepted 18th August 2020.

Address correspondence and reprint request to: Dr. Sultan M. Albugami, College of Medicine, King Saud bin Abdulaziz University for Health Sciences, Riyadh, Kingdom of Saudi Arabia.E-mail: Albaqmi017@ ksau-hs.edu.sa

ORCID ID: https://orcid.org/0000-0003-2635-5590 
$\mathrm{H}^{\mathrm{s}}$ ydrocephalus is a serious condition in which cerebrospinal fluid (CSF) accumulates and causes the intracranial pressure to increase. The mean global prevalence of hydrocephalus is $85 / 100,000 .{ }^{1}$ The etiology behind hydrocephalus can be divided into 3 causes: an obstruction in the flow of the CSF, a defect in reabsorption ofCSF, or overproduction ofCSF. Increased intracranial pressure has different presentation based on age group. Neonates, for instance, will have bulging of the fontanelles, which can lead to disproportionate head growth if left untreated. In older children and adults, headache, vomiting and drowsiness are considered the typical symptoms. Obstructive hydrocephalus can be treated by inserting a ventriculoperitoneal shunt. This shunt bypasses the obstruction and drains the CSF into the peritoneum causing intracranial pressure to return to normal. Shunt failure can occur in 11-25\% of cases. Failure can be due to obstruction or infection of the shunt. This failure is commonly called a shunt malfunction, and it is generally treated by doing a shunt revision. ${ }^{2,3}$

It is challenging to diagnose a shunt malfunction based on the clinical manifestation alone, which causes more reliance on imaging modalities. These diagnostic imaging modalities include CT scan and MRI. It is possible to confirm shunt failure if the ventricles appear dilated in imaging, a sign upon which physicians sometimes rely. This increased reliance can be disastrous in some cases because the imaging modalities occasionally reveal normal-sized ventricles while the shunt malfunction is present. ${ }^{4}$ According to a study conducted at Boston Children's Hospital in 2014 in children who had surgery due to shunt malfunction, CT scan was positive in $65.7 \%$ and MRI was positive in $51.4 \%$ of patients. ${ }^{5}$ Another study conducted at Children's Hospital in Birmingham found that $24 \%$ of the imaging results had described the ventricular system as "unchanged", "stable", "normal", "unremarkable", "small", "smaller", "slit", "negative", and "no hydrocephalus". "There is a lack of researches conducted in Saudi Arabia into imagining reliability in shunt malfunction, which had led to a misleading radiological reports in spite of presence of shunt malfunction. So, this study aimed to determine the prevalence of shunt malfunction without change in ventricle size in imaging. It also defined their

Disclosure. Authors have no conflict of interests, and the work was not supported or funded by any drug company. characteristics, which included: the part of the shunt that was malfunctioning, and the clinical presentation. The study also investigated previous visits to the hospital with complaints of increased intracranial pressure with negative radiological findings.

Methods. The study was approved by the Institutional Review Board (IRB) of King Abdullah International Medical Research Center (KAIMRC). It was conducted in the neurosurgery department of the King Abdulaziz Medical City (KAMC), in Riyadh, Saudi Arabia. It was a cross-sectional study of 42 patients, covering the period from June 2015 to May 2019. Included patients were those who had shunt malfunction and underwent shunt revision. For diagnostic accuracy, the study excluded patients with brain tumors, shunt infections, supratentorial extra-axial fluid collections, holoprosencephaly, hydranencephaly, or those in which the distal terminus of the shunt was in a location other than the peritoneum. The collected data were patients' demographics at time of shunt revision,

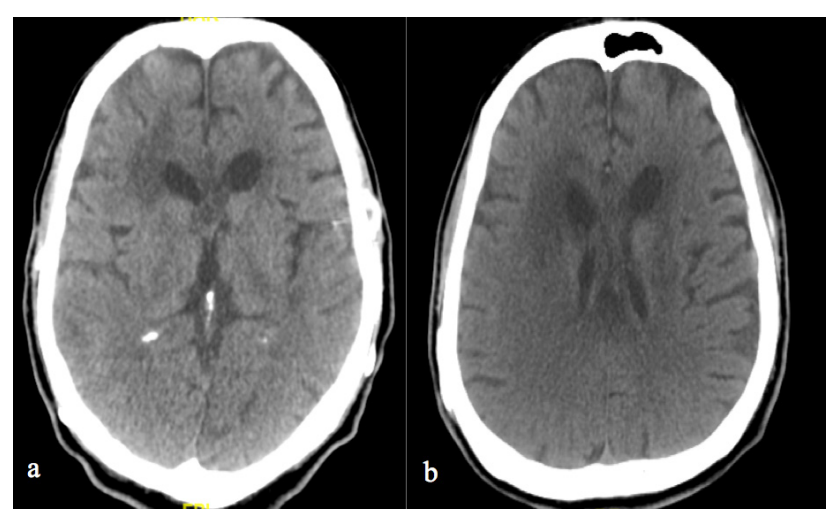

Figure 1 - Non-contrast head CT scan in a patient with a) VP shunt malfunction showed unchanged ventricular size as compared to the previous head CT scan (b).

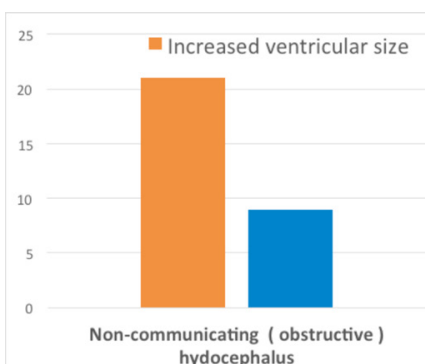

hydocephalus
- Unchanged ventricular size

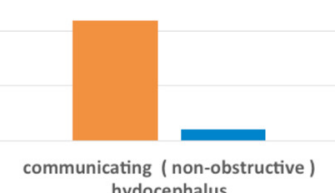

hydocephalus
Figure 2 - Types of hydrocephalus with both increased and unchanged ventricular size. 


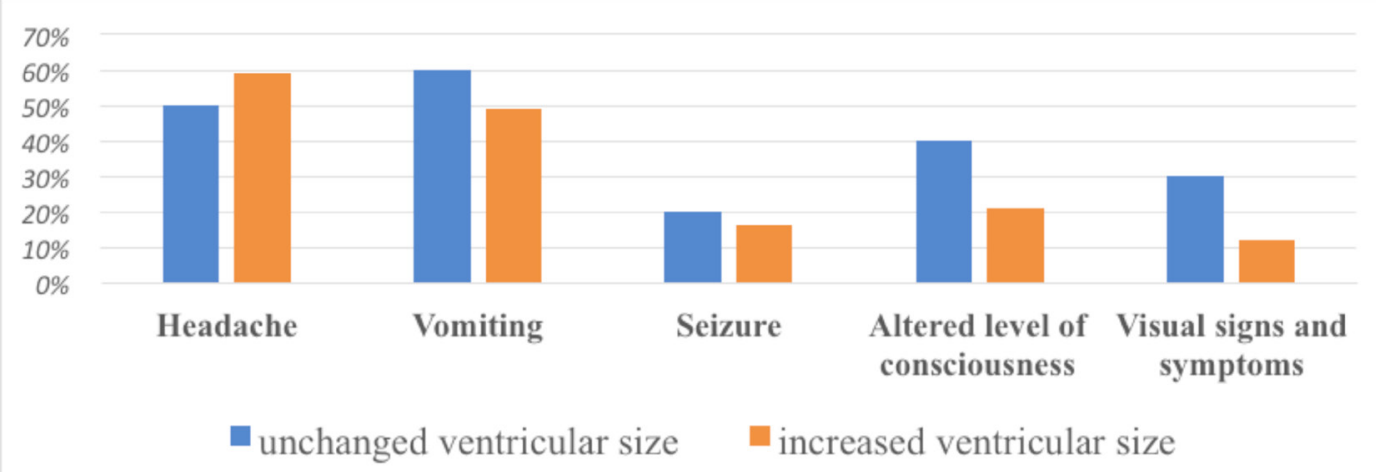

Figure 3 - Signs and symptoms in patients with shunt malfunction both with and without ventricular size change.

Table 1 - Characteristics of the study subjects.

\begin{tabular}{lc}
\hline Characteristic & $\mathbf{n}(\%)$ \\
\hline Age at the time of treatment in years & \\
$\leq 1$ & $4(10)$ \\
$1-10$ & $7(17)$ \\
$11-20$ & $13(31)$ \\
$\geq 21$ & $18(42)$ \\
Gender & \\
Male & $23(55)$ \\
Female & $19(45)$ \\
Types of hydrocephalus & \\
Communicating (non- obstructive) & $12(29)$ \\
Non- communicating (obstructive) & $30(71)$ \\
Determination of ventricular size by radiological Images & \\
No change & $10(24)$ \\
Mildly increased & $8(19)$ \\
Severely increased & $24(57)$ \\
Cause of shunt malfunction & \\
Ventricular catheter occlusion & $14(34)$ \\
Valve malfunction & $10(24)$ \\
peritoneal catheter occlusion & $6(14)$ \\
Combined & $12(28)$ \\
Signs and symptoms & \\
Headache & $24(57)$ \\
Vomiting & $22(53)$ \\
Altered level of consciousness & $11(28)$ \\
Seizure & $7(18)$ \\
Visual signs and symptoms ${ }^{1}$ & $7(18)$ \\
Dizziness & $5(13)$ \\
Gait disturbance & $2(5)$ \\
Dysarthria & $1(3)$ \\
Increased head circumference & $2(5)$ \\
Respiratory failure & $1(3)$ \\
\hline Visual signs and symptoms include papilledema, abducens & $n e r v e ~ p a l s y$ \\
& \\
\hline & \\
& \\
&
\end{tabular}

presenting signs and symptoms, causes and site of shunt malfunction, and the severity change in ventricular size. The size of ventricles was determined by radiological imaging, which included computer tomography scan (CT-scan), magnetic resonance imaging (MRI), and
Table 2 - The association between ventricular size and the most presenting symptoms.

\begin{tabular}{lccc}
\hline Symptom & $\begin{array}{c}\text { Unchanged } \\
\text { ventricular size } \\
\text { n (\%) }\end{array}$ & $\begin{array}{c}\text { Increased } \\
\text { ventricular size }\end{array}$ & $P$-value \\
\hline $\begin{array}{l}\text { Headache } \\
\text { Yes }\end{array}$ & $5(50)$ & $19(60)$ & 0.4 \\
No & $5(50)$ & $13(40)$ & \\
Vomiting & & & \\
Yes & & $6(60)$ & 0.5 \\
No & $4(40)$ & $6(60)$ & \\
Seizure & & & \\
Yes & $2(20)$ & $5(16)$ & 0.5 \\
No & $8(80)$ & $27(84)$ & \\
Altered level of consciousness & & 0.2 \\
Yes & $4(40)$ & $7(22)$ & \\
No & $6(60)$ & $25(78)$ & \\
\hline
\end{tabular}

ultrasound (US). Statistical analysis was carried out using SPSS version 23. Categorical data were presented as frequencies and percentages. Numerical data were presented as means and standard deviation. A chi-square test was used to analyze the associations. P-value less than or equal to 0.05 was considered statistically significant.

Results. A total of 42 patients who underwent a shunt revision during the period from June 2015 to May 2019 were included in this study. The CT scans and MRIs showed no ventricular size change compared with the previous images in $10(24 \%)$ of patients (Figure 1), mild enlargement in $8(19 \%)$ patients, and obvious ventricular size enlargement in $24(57 \%)$ patients. Table 1 shows characteristics of the study population. The mean age at diagnosis was $22 \pm 16.7$ years. In terms of gender, $55 \%$ 
of the patients were male, $45 \%$ were female. Regarding causes, ventricular catheter occlusion was present in 14 (34\%) patients, valve-related malfunction was present in $10(24 \%)$ patients, and peritoneal catheter occlusion was present in $6(14 \%)$ patients, and $12(28 \%)$ cases had some combination of the previous causes. Figure 2 shows Types of hydrocephalus with both increased and unchanged ventricular size. Signs and symptoms in patients with shunt malfunction both with and without ventricular size change are shown in Figure 3. The analysis of the association between ventricular size and headache was statistically insignificant $(p=0.4)$. Also, there was no statistically significant difference in vomiting, seizure, confusion, and loss of consciousness between patients with unchanged ventricular size and those with increased ventricular size (Table 2).

Discussion. It is crucial to detect shunt malfunction cases because missed diagnoses may result in disastrous complications such as permanent neurologic injury or death. ${ }^{5}$ For diagnosis of shunt malfunction, CT scan and MRI both have similar sensitivity, specificity and accuracy rates. ${ }^{6-8}$ Occasionally, neurosurgeons observe shunt malfunction without ventricular dilatation on imaging studies. This study reported $24 \%$ of 42 patients presented with shunt malfunction without ventricular size changes. Other studies reported similar findings; Iskandar et $\mathrm{al}^{9}$ reported $11 \%$ of cases out of 68 patients having a shunt malfunction without ventricular size changes compared to the previous images with an age range from 1 month to 26 years. Amacher et al ${ }^{10}$ reported $4.3 \%$ of 23 patients with $0-17$ age range. McNatt et al ${ }^{11}$ reported $9 \%$ of 177 patients. Sellin et $\mathrm{al}^{12}$ reported $10 \%$ of 42 patients. Mater et $\mathrm{al}^{13}$ reported $10.6 \%$ of 386 patients. Miller et a ${ }^{14}$ reported $27.4 \%$ of 155 patients who had no CT changes and needed shunt revisions. For only pediatric age group patients, Sean et al. reported $9 \%$ of 177 patients with median age of 7.2 years. ${ }^{15}$

All of the mentioned reports suggest that imaging studies are not totally reliable for definitive diagnosis of shunt malfunction because some patients may have a normal or small ventricular size despite the presence of shunt malfunction. Invasive monitoring of intracranial pressure in these groups of patients is also insufficient to make a definitive diagnosis. ${ }^{10-11}$ This study suggests that the safest approach to take upon suspicion of shunt malfunction and for deciding whether surgical intervention is necessary is primarily evaluating patients' clinical signs and symptoms.

Even with ventricular dilation on CT scan, definitive diagnosis of shunt failure cannot be made as ventricular shunt pseudo failure (chronic constipation) can be the actual cause of dilatation thus shunt revision may not be needed and only releasing of bowel content is enough to normalize ventricular dilatation. ${ }^{16}$

One of the risk factors associated with low compliance ventricles is childhood ventriculitis. Small-sized ventricles in shunt malfunction could be due to one or more causes, including: poor compliance, intermittent shunt malfunction, slit ventricle syndrome, CSF leak (through skin incisions, sub glial collections, or the formation or enlargement of a syringohydromyelia), and the possibility that the ventricles were smaller in the past and that what is now observable is in fact large for that particular patient.

In order not to miss an enlarged ventricle that appears normal and to accurately perceive changes, the imaging studies taken when patients present with symptoms of shunt malfunction should be compared with previous imaging studies in which the patient was normal. ${ }^{17}$

A radiology report might be misleading for diagnosing shunt malfunction; therefore, radiologists should be aware and notify neurosurgeons of that fact. Evaluation of shunts by non-specialists who tend to rely on the radiologist's report like emergency physicians, family physicians or pediatricians puts patients at greater risk of misdiagnosing malfunction and of developing fatal complications by sending them home without appropriate management. ${ }^{4,9}$ It is possible to carry out alternatives for malfunction detection other than CT-scan and MRI. These are a shunt tap, shunt patency, intracranial pressure monitoring, long in-hospital observation, and surgical exploration. However, this study suggests that the final determination of shunt functionality should be through surgical exploration.

Ventricles with low compliance have limited ventricular dilation capability. Some authors stated that patients in this situation are at greater risk to encounter acute neurological deterioration with shunt malfunction. ${ }^{14,18}$ However, our results showed no significant association. Single-centered experience and low sample size are limitations of this study. We recommend further multi-center studies on larger groups of patients.

In conclusion, out of 42 cases, we observed $24 \%$ of shunt malfunctions without ventricular dilatation on CT or MR scans. Therefore, detection of shunt malfunction by imaging studies is unreliable. Neurosurgeons should consider this phenomenon when encountering symptomatic patients and for their decision of surgical 
intervention when scans' images reveal no change in ventricular size.

\section{References}

1. Isaacs AM, Riva-Cambrin J, Yavin D, Hockley A, Pringsheim TM, Jette N, et al. Age-specific global epidemiology of hydrocephalus: Systematic review, metanalysis and global birth surveillance. PLoS One 2018; 13: e0204926.

2. Schwamb R, Dalpiaz A, Miao Y, Gonka J, Khan SA. Clinical manifestations of hydrocephalus: a review. Neurol Clin Neurosci 2014; 2: 173-177.

3. Venkataramana NK. Hydrocephalus Indian scenario - A review. J Pediatr Neurosci 2011; 6: S11-S22.

4. Paff M, Alexandeu-Abrams D, Muhonen M, Loudon W. Ventriculoperitoneal shunt complications: A review. Interdiscip Neurosurg 2018; S13: 66-70.

5. Boyle TP, Paldino MJ, Kimia AA, Fitz BM, Madsen JR, Monuteaux MC, et al. Comparison of rapid cranial MRI to CT for ventricular shunt malfunction. Pediatrics 2014; 134 : e47-e54.

6. Yue EL, Meckler GD, Fleischman RJ, Selden NR, Bardo DM, O'Connor AK, et al. Test characteristics of quick brain MRI for shunt evaluation in children: an alternative modality to avoid radiation. J Neurosurg Pediatr 2015; 15: 420-426.

7. Boyle TP, Paldino MJ, Kimia AA, Fitz BM, Madsen JR, Monuteaux MC, et al. Comparison of rapid cranial MRI to CT for ventricular shunt malfunction. Pediatrics 2014; 134: e47-e54.

8. Sato D, Takami H, Teranishi Y, Ishikawa O, Saito N. Pseudofailure of ventriculo-peritoneal shunt due to drugresistant constipation, dramatically responsive to rectal tube placement; pitfall case series of avoidable shunt revision. World Neurosurg 2020; 141: 131-136.
9. Iskandar BJ, McLaughlin C, Mapstone TB, Grabb PA, Oakes WJ. Pitfalls in the Diagnosis of Ventricular Shunt Dysfunction: Radiology Reports and Ventricular Size. Pediatrics 1998; 101: 1031-1036.

10. Amacher AL, Spence JD. Spectrum of benign intracranial hypertension in children and adolescents. Child Nerv Syst 1985; 1: 81-86

11. McNatt SA, Kim A, Hohuan D, Krieger M, McComb JG. Pediatric shunt malfunction without ventricular dilatation. Pediatr Neurosurg 2008; 44: 128-132.

12. Sellin JN, Cherian J, Barry JM, Ryan SL, Luerssen TG, Jea A. Utility of computed tomography or magnetic resonance imaging evaluation of ventricular morphology in suspected cerebrospinal fluid shunt malfunction. J Neurosurg Pediatr 2014; 14: 160-166.

13. Mater A, Shroff M, Al-Farsi S, Drake J, Goldman RD. Test characteristics of neuroimaging in the emergency department evaluation of children for cerebrospinal fluid shunt malfunction. CJEM 2008; 10: 131-135.

14. Miller JP, Fulop SC, Dashti SR, Robinson S, Cohen AR. Rethinking the indications for the ventriculoperitoneal shunt tap. J Neurosurg Pediatr 2008; 1: 435-438.

15. McNatt SA, Kim A, Hohuan D, Krieger M, McComb JG. Pediatric Shunt Malfunction without Ventricular Dilatation. Pediatr Neurosurg 2008; 44: 128-132.

16. Morais BA, Cardeal DD, Andrade FG, Paiva WS, Matushita H, Teixeira MJ. Reversible ventriculoperitoneal shunt dysfunction and chronic constipation: case report. J Neurosurg Pediatr 2018; 22: 147-150.

17. Shapiro K, Fried A. Pressure-volume relationships in shuntdependent childhood hydrocephalus. J Neurosurg 1986; 64: 390-396.

18. Engel M, Carmel PW, Chutorian AM. Increased intraventricular pressure without ventriculomegaly in children with shunts: "normal volume" hydrocephalus. Neurosurgery 1979; 5: 549-552.

\section{Ethical Consent}

All manuscripts reporting the results of experimental investigations involving human subjects should include a statement confirming that informed consent was obtained from each subject or subject's guardian, after receiving approval of the experimental protocol by a local human ethics committee, or institutional review board. When reporting experiments on animals, authors should indicate whether the institutional and national guide for the care and use of laboratory animals was followed. 